\title{
JUEGOS EN EL SALÓN DE CLASE: EL MERCADO DE LOS LIMONES
}

\author{
Luis Alejandro Palacio Garcia* \\ Isabel Saravia Martínez** \\ Maria Alejandra Vesga Cediel****
}

$\mathrm{L}$ a microeconomía es una parte esencial de la ciencia económica que estudia el comportamiento de las personas en diferentes estructuras de mercado y el efecto que tienen las decisiones de los gobiernos sobre el bienestar de la sociedad. Por su parte, la teoría de juegos es un área de la matemática aplicada que utiliza modelos para estudiar interacciones en estructuras formalizadas de incentivos. Sus investigadores estudian las estrategias óptimas, así como el comportamiento previsto y observado de individuos en situaciones bien delimitadas. Aunque estas asignaturas son centrales en la formación de los economistas, indudablemente la enseñanza de la microeconomía implica la exposición y explicación de conceptos teóricos abstractos que pueden resultar difíciles de entender y, por tanto, pueden menoscabar el proceso de aprendizaje. Al abordar estas falencias pedagógicas, los juegos en el salón de clase (Holt, 1996) pueden permitir una mayor apropiación de los conceptos, complementando las clases tradicionales con la metodología de la economía experimental.

Como caso concreto de estudio, el juego del mercado de los limones busca explicar el comportamiento de los agentes en un contexto

* PhD en Economía y Magíster en Ciencias Económicas. Profesor de la Escuela de Economía y Administración, Facultad de Ciencias Humanas, Universidad Industrial de Santander. Investigador del grupo EMAR. [lpalagar@uis.edu.co].

** Economista. Investigadora del grupo EMAR. Universidad Industrial de Santander. [isaa9323@gmail.com].

*** Economista. Investigadora del grupo emar. Universidad Industrial de Santander. [alejandra.vesga93@gmail.com]

Fecha de recepción: 21-09-2016, fecha de modificación: 20-01-2017, fecha de aceptación: 15-04-2017. Sugerencia de citación: Palacio, L.; I. Saravia y M. Vesga. "Juegos en el salón de clase: el mercado de los limones" Revista de Economia Institucional 19, 36, 2017, pp. 291-311. DOI: https://doi.org/10.18601/01245996.v19n36.11 
de negociación que se caracteriza por sus asimetrías de información precontractual (Holt y Sherman, 1999). En particular, el vendedor está mejor informado sobre la calidad del bien que desea transar, poniendo en posición desventajosa al comprador. En el artículo pionero de Akerlof (1970), un "limón" hace referencia a un carro usado de mala calidad. Como el comprador no puede observar la calidad del bien que se le of rece, este debe ser muy cuidadoso, de lo contrario podría pagar un precio muy alto por un limón.

En los cursos de microeconomía usualmente se enseña que en el mercado los precios se determinan por la interacción de la oferta y la demanda, alcanzando unas cantidades de equilibrio eficientes. Este modelo se basa en los supuestos de competencia perfecta y racionalidad individual; es decir, no existen externalidades ni costos de transacción y los agentes son precio aceptantes y maximizadores de su función objetivo. Sin embargo, Akerlof (1970) demostró que basta con relajar el supuesto de información perfecta para alcanzar, en equilibrio, una asignación de recursos no óptima en el sentido de Pareto. En efecto, las investigaciones por las que George Akerlof, Michael Spence y Joseph Stiglitz fueron premiados en 2001 con el Nobel en economía forman parte de un programa de investigación que muestra que la economía de la información representa un cambio fundamental en el paradigma vigente de la ciencia económica (Stiglitz, 2002).

El objetivo de este artículo es presentar el proceso de diseño y validación de un protocolo experimental que permite a los estudiantes entender y debatir el efecto de los fallos de mercado relacionados con la selección adversa. En este sentido, el juego del mercado de los limones permite a los estudiantes observar hasta qué punto los bienes de buena calidad pueden ser expulsados del mercado por los bienes de mala calidad (limones). Gracias a la combinación de la teoría de juegos y la metodología experimental, los estudiantes se aproximarán de una manera más intuitiva a las predicciones teóricas formuladas por este modelo, e incluso pueden identificar la relevancia de los supuestos y las aplicaciones a situaciones cotidianas.

Según Emerson (2014), la literatura relacionada con juegos en el salón de clase se divide en dos categorías generales. E1 primer grupo implica nuevos experimentos en el aula que se encuentran en desarro1lo. Los diseñadores y los defensores de los experimentos en el aula, como los de cualquier otro enfoque de aprendizaje activo, basan sus aportes sobre las mejoras en los resultados de los estudiantes asociados con el uso de la técnica. Los juegos que abordan el problema de 
las asimetrías de información diseñados por Holt y Sherman (1999), Capra et al. (2000) o Wolf y Myerscough (2007) son buenos ejemplos de esta categoría. Por su parte, los artículos de investigación que tratan de verificar la eficacia de los experimentos en el aula constituyen la segunda categoría. Para el caso colombiano se encuentran los aportes de García (2004), Salazar (2010) y Muñoz, Aparicio y Mesa (2011). Una discusión más amplia de la investigación sobre enseñanza de la economía se encuentra en Sarnikar (2015).

Este artículo aporta a la literatura sobre diseño y validación de juegos en el salón de clase a partir de la experiencia acumulada por el grupo de investigación Estudios en Microeconomía Aplicada y Regulación (EMAR). Es importante aclarar que el protocolo experimental del juego del mercado de los limones se diseñó como parte del trabajo de grado de Saravia y Vesga (2015). Este trabajo de grado fue una pasantía de investigación en el marco del proyecto de investigación titulado "Análisis de las decisiones individuales en contextos de negociación. Aportes teórico y experimental”. Además, esta línea de investigación se consolidó en el proyecto "Juegos en el salón de clase: una aplicación de los experimentos económicos a la enseñanza de la microeconomía”, el cual fue financiado como un proyecto de articulación de la investigación y la extensión con la docencia. En este sentido, para ejecutar los proyectos se conformó un equipo de trabajo con estudiantes e investigadores del grupo EMAR y actualmente se está consolidando el Laboratorio de Economía Experimental del grupo EMAR (EMAR-LAB) ${ }^{1}$.

Por lo tanto, el juego del mercado de los limones que aquí se presenta busca documentar y compartir las experiencias y aprendizajes obtenidos por el grupo de investigación, proponiendo una reflexión más amplia sobre la relevancia de las técnicas experimentales en la enseñanza de la economía. El propósito es difundir esta nueva metodología, mostrando sus ventajas, alcances y limitaciones, y explicando de forma sencilla las nociones básicas subyacentes. En particular, la discusión se centrará en las ventajas de adaptar una sala de cómputo para cumplir las funciones de laboratorio; es decir, se argumentará que el uso de computadores permite sistematizar las decisiones para contrastar el efecto de diferentes variables de interés.

${ }^{1}$ En total se realizaron 10 trabajos de grado en el marco de estos proyectos, cada uno buscando diseñar un protocolo para abordar diferentes problemas teóricos. Los 10 juegos realizados fueron: 1) Bienes públicos, 2) Mercados prohibidos, 3) Juego del conflicto, 4) Juego de la confianza, 5) Dilema del viajero, 6) Juego del ultimátum, 7) Juego de la guerra, 8) Mercado de los limones, 9) Modelo de Cournot, y 10) Teorema de Coase. 
Para cumplir este propósito, el texto se organiza de la siguiente manera. En la segunda sección se abordan las características generales que definen los juegos en el salón de clase, basando la argumentación en la experiencia acumulada por el grupo EMAR. Posteriormente se presenta el protocolo experimental del mercado de los limones, programado por medio del software $z$-Tree (Fischbacher, 2007). Finalmente se esbozan las principales conclusiones obtenidas del trabajo.

\section{METODOLOGÍA EXPERIMENTAL Y ENSEÑANZA DE LA ECONOMÍA}

Las decisiones económicas tienen serias implicaciones reales en los mercados y, sobre todo, en el bienestar de las personas. Por esta razón es fundamental que la formación académica de los futuros economistas garantice que el aprendizaje esté orientado a dar solución a los problemas de la sociedad. No obstante, cuando la comprensión de la realidad parte del entendimiento de las formalidades matemáticas, los estudiantes pueden ver el proceso de enseñanza-aprendizaje como una experiencia difícil y poco agradable (Muñoz, Aparicio y Mesa, 2011).

De acuerdo con Becker y Watts (2001), esta problemática obedece a que el estudiante no es un actor activo de su propio proceso de aprendizaje. En Colombia, por ejemplo, los métodos de enseñanza más frecuentes son las exposiciones magistrales de "tiza y tablero" y las presentaciones de Power Point, ambas estructuradas alrededor de los contenidos puntuales de los libros de texto (Lora y Ñopo, 2009). Estas metodologías son poco interactivas, y por lo tanto, tienden a disminuir el interés de los estudiantes por las clases. Lo anterior no significa que los métodos tradicionales sean obsoletos, sino que deben combinarse con técnicas alternativas para hacer más eficiente el proceso de enseñanza-aprendizaje.

En este sentido, se puede afirmar que la economía experimental cuenta con valiosas herramientas que, usadas apropiadamente con fines pedagógicos, pueden ayudar a llenar este vacío, llevando de una forma interactiva a que los estudiantes encuentren la relevancia de los temas vistos en clase. Esto se debe a que la puesta en práctica de los conocimientos obtenidos y la comparación de estos con las predicciones teóricas permite una comprensión más rápida e intuitiva. Todo esto gracias a la capacidad del experimentador de controlar el entorno y recrear los ambientes teóricos en la sala de cómputo adaptada como laboratorio. Adicionalmente, el uso de computadores hace que todas las decisiones se puedan sistematizar para contrastar el efecto de diferentes variables de interés. 


\section{Juegos en el salón de clase: la experiencia del grupo emar}

Un experimento económico es una recreación de una situación en la que un grupo de individuos toma decisiones que se pueden contrastar contra lo que predice la teoría. Más concretamente, un experimento tiene tres ingredientes básicos: 1) un entorno, 2) unas instituciones formales y 3 ) el comportamiento específico de cada individuo (Palacio y Parra, 2012). En términos generales, en un experimento se busca contrastar empíricamente las predicciones de un modelo formal, aislando los efectos de diferentes variables; es decir, determinar si la teoría es realmente correcta bajo condiciones ceteris paribus (Hey, 1991)2.

Aunque la economía experimental es el referente metodológico, es importante hacer una aclaración conceptual, pues los juegos en el salón de clase buscan la apropiación social del conocimiento, no pretenden validar o refutar una teoría. En este sentido, Holt (1996; 2007) define los juegos en el salón de clase como un ejercicio pedagógico que aumenta el interés de los estudiantes en la teoría económica. Este enfoque busca complementar las exposiciones magistrales y los libros de texto con juegos en los que los estudiantes toman decisiones e interactúan.

Si bien es posible realizar actividades lúdicas solo con papel y lápiz en un aula convencional, es conveniente pensar que los juegos se realicen en un laboratorio de economía experimental; es decir, en un espacio físico adaptado con fines de investigación. La ventaja de consolidar un laboratorio es facilitar los procedimientos logísticos y de apoyo para que los diferentes investigadores puedan probar sus hipótesis, divulgar sus resultados de investigación y realizar sus clases cuando utilicen estas metodologías de aprendizaje activo. Cada una de las fases del proyecto se facilita en la medida en que se gana experiencia en los procedimientos, se logra dividir el trabajo y se aumenta la reputación por las buenas prácticas.

Siguiendo lo planteado por Palacio y Parra (2012), los juegos en el salón de clase buscan recrear una situación interesante en términos teóricos en un ambiente de laboratorio. El investigador logra controlar cada aspecto aislando aquellos fenómenos que en la realidad son difíciles de estudiar, y que por lo general son difíciles de entender en las clases magistrales. No se pretende replicar de manera exacta una

\footnotetext{
${ }^{2}$ En la actualidad se ha ampliado considerablemente la literatura sobre economía experimental, lo que se refleja en diversos libros de texto como el de Friedman y Cassar (2005), donde se enseñan paso a paso los requerimientos básicos necesarios para hacer un experimento. Del mismo modo, Brañas (2011), Bergstrom y Miller (2000), Hey (1991) y Montenegro (1995) muestran la amplia gama de aplicaciones de esta metodología.
}

Revista de Economía Institucional, vol. i9, n.o 36, Primer semestre/2oi7, Pp. 29i-3ii 
situación del mundo real; se busca aprender algo útil, cuestionar lo visto en clase y fomentar un debate enriquecedor. La ventaja de usar los computadores es que permite recrear los modelos teóricos; es decir, los estudiantes se enfrentan a los incentivos económicos, interactúan con sus compañeros en diferentes roles y toman decisiones buscando ganar puntos dentro del juego.

Una investigación de laboratorio, típicamente, procede en cuatro fases: 1) se determina el modelo a contrastar y el entorno adecuado; 2) se realizan unas sesiones exploratorias para verificar si el protocolo cumple con lo esperado; 3) se obtienen los datos de la investigación realizando varias sesiones; 4 ) se realizan varias sesiones de seguimiento para proporcionar evidencia definitiva (Friedman y Sunder, 1994). En términos generales, para poder diseñar y validar el protocolo de un juego en el salón de clase se debe seguir este procedimiento, pero adaptando algunas fases de la investigación a las particularidades y objetivos planteados. A continuación se describen las generalidades de los juegos que ha realizado el grupo EMAr. Con esto se busca compartir la experiencia acumulada, teniendo claro que los procedimientos siempre se pueden mejorar y adaptar a las necesidades de cada población objetivo.

Una vez se tiene la programación del juego diseñado por los investigadores, o una adaptación de los juegos que se encuentran en la literatura, se procede con su validación. Para esto se realiza una convocatoria abierta a los estudiantes que deseen participar, quienes no tienen información específica sobre las particularidades del mismo. Cuando inicia la sesión, los organizadores les asignan un computador privado y se les presentan unas instrucciones en cada pantalla individual. Los participantes se conectan al experimento, leen las instrucciones, responden una serie de preguntas para garantizar que entendieron el ejercicio propuesto y se les comunica que han sido emparejados de forma anónima con otra persona de la sala, cuya identidad nunca les será revelada. Luego deben interactuar en una serie de periodos, pasando por varios tratamientos de acuerdo al diseño del juego. Esto garantiza que los estudiantes se sitúen en una representación esquemática de una situación económica en la que tienen un papel específico: empresas, consumidores, inversionistas, trabajadores, etc.

De acuerdo con Smith (2005), los experimentos han demostrado que el contexto social puede ser importante en el comportamiento que observamos. Por lo tanto, lo que se busca con las instrucciones es facilitar un contexto de partida que evite palabras emotivas que 
pudieran desencadenar significados no buscados por el experimentador. Los participantes deben entender que se trata de una situación con un cierto carácter artificial en la que las únicas reglas explícitas vienen dadas por las instrucciones y donde su objetivo individual es ganar la mayor cantidad de puntos.

Cada participante puede escoger entre varias opciones y cada una de estas implica ciertos pagos o puntos, dependiendo de la decisión del participante en cuestión y también de lo que hagan los demás. Luego, los organizadores propician un debate académico resaltando las decisiones tomadas y contrastándolas con las predicciones teóricas. Finalmente se aplica un instrumento de evaluación a los estudiantes que permite medir el efecto del juego en la comprensión y el aprendizaje de los conceptos microeconómicos. Los organizadores observan y registran las decisiones tomadas por los participantes y emplean estos datos como fuente de información sobre el problema económico que quieren recrear (Brandts, 2007), permitiendo ajustar el protocolo cuando se evidencie que se pueden implementar mejoras. Si se cumple a cabalidad con los objetivos, toda la experiencia se documenta para que pueda ser replicada como estrategia pedagógica en las clases que normalmente se programan en la universidad.

\section{Consideraciones éticas}

Lo que diferencia a los experimentos de otros métodos económicos es la interacción de las personas en entornos controlados. Este tipo de investigación es relevante cuando el comportamiento humano ayuda a arrojar nueva luz en uno de los muchos pasillos de la economía que se han quedado en la oscuridad por otros métodos (Friedman y Sunder, 1994). Como se ha expuesto, en los juegos en el salón de clase los estudiantes de economía son sujetos activos, sea como participantes o como pasantes en la investigación. Por lo tanto, para garantizar buenas prácticas y alcanzar los objetivos pedagógicos, todo el equipo de investigadores debe seguir unos protocolos muy estrictos, guiándose siempre por principios éticos adaptados para este tipo de intervenciones.

Previo a realizar la investigación, en la Universidad Industrial de Santander se requiere el aval del Comité de Ética en Investigación Científica-CEINCI, que implica cumplir con los lineamientos para los proyectos que involucren aspectos sociales y empresariales. Lo que se busca es la protección de los sujetos de investigación, las comunidades, los grupos vulnerables y la institucionalidad, conservando en todo momento la confidencialidad de las distintas fuentes primarias 
y secundarias. De igual manera, la protección de los investigadores y colaboradores. A continuación se describen los principios éticos que deben seguir los juegos en el salón de clase en la Universidad Industrial de Santander cuando se está trabajando en el marco de un proyecto de investigación, no sin antes advertir que solo se busca tener elementos de juicio que permitan la discusión sobre buenas prácticas cuando se piensa en innovación pedagógica.

Respeto y autonomía: en todo momento se deja claro a los estudiantes que su participación en los juegos es voluntaria y que tiene fines académicos. Para garantizar la voluntariedad, al ingresar a la sala experimental se solicitará el consentimiento informado escrito de cada participante. Los participantes tienen derecho a retirarse del estudio en cualquier momento y pueden negarse a tomar decisiones experimentales cuando no se sientan cómodos. En ningún caso su retiro o negativa a participar en la actividad tendrán consecuencias en la nota del curso o darán lugar a represalias en las clases.

Beneficencia - no maleficencia: la participación en cada juego se limitará a interactuar con un grupo de personas y tomar decisiones individuales en un ambiente computarizado controlado. En tal sentido, el nivel de riesgo al que se verán expuestos los estudiantes es mínimo, por lo que en ningún momento su integridad psicofísica se verá comprometida. Esto implica que la probabilidad y la magnitud del malestar previsto no son superiores a los que se encuentran en la vida diaria o en el curso de un examen académico normal. Lo anterior significa que los beneficios potenciales (aportes a su formación académica) para cada participante serán mayores a los riesgos que correrá. De otra parte, cabe añadir que el diseño metodológico propuesto es adecuado y los investigadores son profesionales competentes para implementar exitosamente la metodología de estudio y salvaguardar el bienestar de los alumnos.

Justicia: ningún estudiante que participe en los juegos será objeto de discriminación por razones étnicas, sexuales, creencias religiosas o cualquier otra circunstancia. La participación es totalmente gratuita y no requiere ninguna preparación previa. Del mismo modo, es preciso aclarar que en ningún caso los estudiantes que participen en los juegos recibirán beneficios en sus calificaciones como premio por su participación, y que de ninguna manera el hecho de negarse a participar repercutirá negativamente en las notas del curso.

Confidencialidad: la información que suministren los estudiantes se mantendrá bajo estricta confidencialidad. E1 software del sistema llevará un registro detallado de todas las decisiones que se tomen 
en cada juego y generará una base de datos segura. E1 anonimato es garantizado, pues ni siquiera el personal profesional conocerá la identidad de quien ha tomado cada decisión. Los resultados agregados serán discutidos en el grupo con fines puramente académicos, pero en ningún caso se utilizará el nombre o cualquier otra información que pueda identificar personalmente a cualquiera de los participantes. En todo caso se cumplirá con lo reglamentado en la Ley estatutaria 1581 de 2012 y la Resolución de rectoría uis no. 1227 de agosto 22 de 2013, sobre el tratamiento de datos personales y el derecho de habeas data de todos los estudiantes que participen en los juegos de salón de clase. En tal sentido, se reconoce el derecho individual de acceder, actualizar o suprimir la información suministrada, así como el derecho de revocar la autorización otorgada para el tratamiento de la misma.

Antes de terminar esta sección es importante insistir en que los juegos en el salón de clase se diseñan como parte de un proceso de investigación-acción participativa, donde los estudiantes no se consideran el "objeto de estudio" sino el "sujeto protagonista"; es decir, busca entender el proceso de enseñanza-aprendizaje al tratar de cambiarlo colaborativa y reflexivamente. En este sentido, lo que se pide a los estudiantes es que lean unas instrucciones en el computador y tomen unas decisiones que afectan los puntos que ellos y los otros participantes obtienen en el juego. El objetivo de cada estudiante es ganar la mayor cantidad de puntos posible. Como consecuencia de su interacción y gracias al cambio de tratamientos, los estudiantes entienden que las consecuencias de sus decisiones se ven afectadas por diferentes variables de interés desde el punto de vista teórico. Por último, se propicia un debate enriquecedor motivado por la propia experiencia. Por lo anteriormente expuesto se considera que el riego asociado a los juegos en el salón de clase es mínimo.

Una vez se considera que el protocolo está suficientemente validado, el proceso de investigación da paso a la aplicación de los resultados en las clases tradicionales y en diferentes espacios de difusión. En otras palabras, dado que los estudiantes han sido sujetos activos, como participantes en las sesiones, pero también como parte del equipo de investigación, el resultado final es un protocolo experimental adaptado y validado en un contexto específico. En la siguiente sección se describe el caso concreto del mercado de los limones.

\section{EL MERCADO DE LOS LIMONES}

Transar, negociar y llegar a acuerdos hace parte de muchas situaciones cotidianas. Dentro de esta interacción, los individuos se enfrentan a 
fallos en la información que generan ineficiencias. En otras palabras, debido a las imperfecciones en la información, los agentes económicos intentarán tomar ventaja cuando están mejor informados, pero el resultado final es que muchas transacciones mutuamente beneficiosas no se llevarán a cabo. Esta idea fue originalmente expuesta por Akerlof (1970), haciendo célebre entre los economistas "el mercado de los limones" como un caso representativo del problema de la selección adversa. Este modelo es presentado en algunos cursos introductorios e intermedios de microeconomía, tales como organización industrial, regulación, teoría de la firma, análisis económico del derecho, teoría de juegos y economía experimental.

Tomando como base el protocolo diseñado por Holt y Sherman $(1999)^{3}$, el juego que se explica a continuación busca contrastar las decisiones de los agentes económicos bajo contextos de negociación con información simétrica y asimétrica. La idea general del juego es la siguiente: el computador organiza a los participantes por parejas de forma aleatoria, asignándole a cada estudiante un rol, vendedor o comprador. Los estudiantes vendedores escogen la calidad y el precio para su producto, los compradores deciden si aceptan o rechazan la propuesta. Con información simétrica, el precio y la calidad son mutuamente conocidos, por lo que los compradores eligen en igualdad de condiciones en términos de información. En este contexto, que será la línea base, los precios y la calidad ofrecida en el mercado rápidamente alcanzan niveles eficientes que maximizan el excedente total.

Por el contrario, sin información simétrica los compradores solo observan el precio (no la calidad). Los vendedores siguen escogiendo el precio y la calidad, pero están mejor informados que los compradores sobre la calidad del bien que se ofrece. Con esta pequeña diferencia como tratamiento se observa que el precio y la calidad caen a niveles ineficientemente bajos. Estos cambios en el comportamiento observado pueden estimular una amplia discusión sobre las asimetrías de información, el fracaso del mercado y algunos remedios tales como estándares de calidad y garantías.

\section{Información simétrica: línea base}

En el juego existen dos tipos de participantes: vendedor y comprador. El juego se desarrolla en tres etapas. En la primera etapa el vendedor

Como se explicó en la sección anterior, el protocolo que aquí se presenta es el resultado del proceso de diseño y validación realizado por el grupo EMAR. En este sentido, algunos elementos del diseño Holt y Sherman (1999) se adaptaron y modificaron para realizar las sesiones en el laboratorio. 
determina la calidad del bien y fija un precio de venta. Posteriormente, en la etapa 2 el comprador observa la calidad y el precio propuestos por el vendedor y debe decidir si desea o no comprar el bien. Por último, se informa a los jugadores los pagos obtenidos en función de las decisiones.

Con respecto a los pagos, el bien puede ser de diferentes calidades, ordenadas desde la menor calidad (1) hasta la mejor calidad (5). El costo de producción del vendedor y el valor que le da el comprador al bien dependen de la calidad, como se puede ver en la matriz costovalor cuadro 1 a diferencia del diseño de Holt y Sherman (1999), la información contenida en la matriz costo-valor es conocimiento común.

Cuadro 1

Matriz costo-valor

\begin{tabular}{lccccc}
\hline Calidad & 1 & 2 & 3 & 4 & 5 \\
\hline Costo & 1.000 & 2.000 & 3.000 & 4.000 & 5.000 \\
Valor & 2.000 & 4.000 & 6.000 & 8.000 & 10.000 \\
\hline
\end{tabular}

Fuente: elaboración propia.

Los pagos dependerán, por parte del vendedor, de su decisión en cuanto a la calidad y precio del bien; por parte del comprador, de si este acepta o no la propuesta. Con estos elementos, si el comprador acepta la propuesta, los puntos del vendedor se calcularán como el precio menos el costo. Los puntos del comprador se calcularán como el valor menos el precio. Si el comprador no acepta la propuesta del vendedor, los dos participantes obtendrán cero puntos. Considérese el siguiente ejemplo para entender las decisiones y los pagos. Supóngase que el vendedor decide ofrecer un bien de calidad 3. Además, decide que el precio de venta es 4.000. El comprador, por su parte, observa esta propuesta y la acepta. Por lo tanto, el vendedor obtendría 1.000 puntos, la diferencia entre el precio (4.000) y el costo (3.000). El comprador obtendría 2.000 puntos, la diferencia entre el valor (6.000) y el precio (4.000). Para mayor claridad, si en este mismo ejemplo el comprador decide rechazar la propuesta los pagos de los dos jugadores serían 0 puntos.

\section{Tratamientos e hipótesis}

Dados los incentivos que enfrentan los participantes del juego, ¿cuál sería el comportamiento que se espera observar? Para responder esta pregunta es importante calcular el equilibrio perfecto en subjuegos por inducción hacia atrás. Piénsese en la decisión que debe tomar el 
comprador si quiere maximizar sus puntos. La propuesta que observa es una dupla precio/calidad, y se sabe que siempre que el precio sea estrictamente menor que el valor (que depende de la calidad) el comprador aceptará la propuesta, pues de no hacerlo obtendría 0 puntos.

Con esto en mente es posible determinar la mejor respuesta del vendedor. Se sabe que este debe buscar la mayor diferencia entre el precio y el costo. Nótese que la matriz costo-valor está diseñada para que las diferencias entre valor y costo se incrementen cuando aumenta la calidad ${ }^{4}$. Para la calidad 1 la diferencia es de 1.000 puntos y para la calidad 5 es de 5.000 puntos. Por lo tanto, el vendedor debe ofrecer la calidad 5 dado que es donde puede obtener un excedente mayor, siempre y cuando el precio sea menor al valor. Para no complicar la explicación, la propuesta calidad 5 a precio 9.999 le garantizaría al vendedor un pago de 4.999 puntos, y el comprador aceptaría porque es mejor 1 punto a quedarse sin nada.

Esto lleva a la predicción que se quiere contrastar: cuando existe información simétrica se espera que los bienes transados mayoritariamente sean de calidad 5, y por lo tanto el precio debe ser mayor a 5.000. Esto permitiría inferir que el mercado llevaría a las partes a una asignación de recursos óptima en el sentido de Pareto. Con esto en mente, lo más interesante es observar cómo cambia el comportamiento sin información simétrica, es decir, cuando el vendedor está mejor informado sobre la calidad del bien a transar.

Con este propósito, el tratamiento experimental es realizar el juego con una pequeña diferencia: que el comprador solo pueda observar el precio, pero no esté informado sobre la calidad. En términos formales, la etapa 1 del juego sería exactamente igual, y los pagos se calcularían de la misma forma. La diferencia estaría en la información disponible al comprador en la etapa 2 . ¿Esta variación en el juego cambiará el comportamiento de los participantes?

Sin información simétrica la decisión del comprador es más compleja porque se enfrenta a una elección bajo incertidumbre. Para ilustrarlo, supongase que este observa un precio alto, superior a 8.000. Sus pagos serían positivos solo cuando la calidad sea 5 , de lo contrario estaría pagando más de lo que valora el bien. Claramente el comprador debe ser cauto: ante un precio alto lo mejor es rechazar la oferta, con el fin de evitar una transacción desventajosa. La predicción no puede ser más precisa en términos de utilidad esperada porque no

\footnotetext{
${ }^{4}$ Este es otro elemento que se decidió modificar con respecto al diseño propuesto por Holt y Sherman (1999) para hacer más claros los resultados y facilitar el debate con los estudiantes.
} 
es posible calcular objetivamente la probabilidad de estar ante una propuesta de calidad 5.

Siguiendo esta misma lógica, piensese ahora que el comprador observa un precio no tan alto (superior a 6.000 pero inferior a 8.000). En este caso el comprador aceptaría si supiera con certeza que el bien es de calidad 4 o 5 , pero no puede estar seguro de eso. Nuevamente, si acepta y la calidad es 3 o inferior, obtendría pagos negativos. El problema es más complejo cuando el precio es inferior a 5.000: a partir de este punto el comprador estará seguro de que no le pueden estar of reciendo un bien de calidad 5, dado que el vendedor no puede realizar una propuesta que le implique pérdidas (el computador no se lo permite). De este mismo modo, a un precio de 4.000 el comprador tendrá la certeza de que no le están ofreciendo bienes de calidad 4 o 5, y así sucesivamente hasta llegar a un precio entre 1.000 y 2.000 , donde, con toda seguridad, solo le están of reciendo bienes de calidad 1.

En resumen, esta lógica muestra que los bienes de buena calidad pueden ser expulsados del mercado por los "limones". Los vendedores pueden anticipar que las propuestas con precios altos serán rechazadas por los compradores para protegerse de posibles engaños, obligándolos a bajar los precios. Pero, como existen bienes de diferentes calidades, el mecanismo sería una caída en cascada; es decir, es posible que los malos expulsen a los no tan malos, estos a su vez expulsen a los no tan buenos, los cuales expulsen a los buenos, y en tal secuencia de eventos terminarán solo transándose bienes de la peor calidad a precios muy bajos.

\section{Procedimientos experimentales para validar el protocolo}

Se realizaron 4 sesiones en total, dos el 17 y dos el 19 de junio de 2015. El proceso de reclutamiento se hizo los días 9 y 15 de junioa través de un correo electrónico enviado a las personas inscritas en la base de datos del grupo EMAR. La persona que deseaba participar en el juego respondía el correo manifestando su interés y su disponibilidad de tiempo para alguna de las sesiones, e inmediatamente recibía un correo de confirmación que indicaba que había quedado inscrita. En caso de que para la sesión ya no hubiera cupo, se le informaba al interesado. Finalmente, un día antes de la realización del juego se envió un correo de confirmación para recordar el lugar y la hora en la que se inscribió. En total participaron 70 personas, principalmente estudiantes de la Universidad Industrial de Santander. La información general de la convocatoria se describe en el cuadro 2. 
Cuadro 2

Reclutamiento

\begin{tabular}{|c|c|c|c|c|c|c|c|c|}
\hline Sesión & Fecha & Hora & $\begin{array}{c}\text { Correo } \\
\text { reclutamiento }\end{array}$ & $\begin{array}{c}\text { Correo } \\
\text { confirmación } \\
\text { (o no cupo) }\end{array}$ & $\begin{array}{l}\text { Correo } \\
\text { recordatorio }\end{array}$ & $\begin{array}{c}\text { Personas } \\
\text { confirmadas }\end{array}$ & Asistentes & $\begin{array}{c}\text { No } \\
\text { asistentes }\end{array}$ \\
\hline 1 & $\begin{array}{l}\text { Miércoles } \\
17 \text { de } \\
\text { junio }\end{array}$ & $\begin{array}{l}8-10 \\
\text { a.m. }\end{array}$ & $\begin{array}{c}9 \text { y } 15 \\
\text { de junio }\end{array}$ & $\begin{array}{c}9 \text { y } 19 \\
\text { de junio }\end{array}$ & $\begin{array}{c}\text { Martes } 16 \\
\text { de junio }\end{array}$ & 15 & 18 & 2 \\
\hline 2 & $\begin{array}{l}\text { Miércoles } \\
17 \text { de } \\
\text { junio }\end{array}$ & $\begin{array}{l}2-4 \\
\text { p.m. }\end{array}$ & $\begin{array}{c}9 \text { y } 15 \\
\text { de junio }\end{array}$ & $\begin{array}{c}9 \text { y } 19 \\
\text { de junio }\end{array}$ & $\begin{array}{c}\text { Martes } 16 \\
\text { de junio }\end{array}$ & 18 & 16 & 4 \\
\hline 3 & $\begin{array}{l}\text { Viernes } \\
19 \text { de } \\
\text { junio }\end{array}$ & $\begin{array}{c}10-12 \\
\mathrm{~m} .\end{array}$ & $\begin{array}{c}9 \text { y } 15 \\
\text { de junio }\end{array}$ & $\begin{array}{c}9 \text { y } 19 \\
\text { de junio }\end{array}$ & $\begin{array}{c}\text { Jueves } 18 \text { de } \\
\text { junio }\end{array}$ & 20 & 16 & 4 \\
\hline 4 & $\begin{array}{l}\text { Viernes } \\
19 \text { de } \\
\text { junio }\end{array}$ & $\begin{array}{l}4-6 \\
\text { p.m. }\end{array}$ & $\begin{array}{c}9 \text { y } 15 \\
\text { de junio }\end{array}$ & $\begin{array}{c}9 \text { y } 19 \\
\text { de junio }\end{array}$ & $\begin{array}{c}\text { Jueves } 18 \text { de } \\
\text { junio }\end{array}$ & 24 & 20 & 4 \\
\hline
\end{tabular}

Fuente: elaboración propia.

Las sesiones del juego se realizaron en las instalaciones del EMARLAB. Cada sesión consta de 20 periodos, divididos en dos etapas, 10 periodos iniciales y 10 periodos finales. Esto con el fin de que los estudiantes interactuaran en los dos tratamientos, Con y Sin información sobre la calidad. Para controlar el efecto orden y el posible efecto del reinicio, se realizaron sesiones donde en los periodos iniciales se interactuaba con información y luego sin información, y otras sesiones con el orden contrario. Al inicio de cada periodo el computador organiza a los participantes por parejas, determinando aleatoriamente el tipo (vendedor o comprador). De igual forma, las parejas son reasignadas al azar en cada periodo. En la literatura esto se conoce como el protocolo "extraños" y se utiliza para controlar el efecto de la reputación. Para asegurar el anonimato y la confidencialidad se asigna a cada estudiante un número al azar para determinar el computador que cada cual utilizará.

En cada sesión se procede en cinco pasos secuenciales y complementarios que se describen a continuación: 1) Se presentan las instrucciones necesarias para que los estudiantes participen en el juego ${ }^{5}$; 2) Se realizan los diferentes tratamientos experimentales; 3 ) Se explica la teoría económica subyacente y se estudia el paralelismo existente entre el modelo teórico y el diseño experimental; 4) Se propicia un debate grupal en torno a dichos resultados empíricos; 5) Se aplica una encuesta de percepción que permite evidenciar el nivel de satisfacción de los estudiantes por participar en el juego.

5 Las instrucciones se presentaban en cada pantalla individual, por lo que se incorporaron como parte de la programación en $z$-Tree. Para una consulta general, el texto de las instrucciones está disponibles en el blog del grupo emar, Racionalidad Ltda., [https://racionalidadltda.wordpress.com/2016/04/22/el-mercadode-los-limones-instrucciones/] 
Las reglas quedan claramente especificadas en las instrucciones, pero muchas veces surgen dudas. Para asegurar que los participantes han comprendido los elementos principales del juego, se realiza un breve cuestionario, de manera que solo cuando lo responden correctamente podrán empezar a jugar. Básicamente los participantes deben entender cómo se calculan los pagos, aunque siempre se aclara en voz alta que una vez empiece el juego el computador realizará los cálculos automáticamente. Adicionalmente se hacen unas preguntas sobre el protocolo específico: ¿Cuántos vendedores hay en cada pareja? (un vendedor en cada pareja); $\dot{¿}$ Los puntos que tú obtengas dependerán solo de las decisiones que tú tomes? (no, lo que el otro decida afecta mis pagos); ¿Durante el experimento tu pareja será reasignada al azar en cada periodo? (sí, tanto la pareja como el rol cambian aleatoriamente); $L$ Los puntos que obtienen tanto el vendedor como el comprador dependen del costo, el valor y la calidad del bien? (sí, estas variables son claves para calcular los pagos). Por supuesto, el personal de apoyo tiene muy claro que se debe evitar cualquier explicación que pueda revelar las condiciones subyacentes al modelo y los resultados esperados.

Con el fin de motivar a los participantes y buscar, en la medida de lo posible, los requisitos del valor inducido, se explicó que el objetivo en el juego es acumular la mayor cantidad de puntos posible. Además se entregó un premio monetario a los tres participantes con mayor puntaje en cada sesión de la siguiente manera: primer puesto, COP $\$ 25.000$; segundo puesto, COP $\$ 15.000$; tercer puesto, COP $\$ 10.000$. Este esquema de incentivos por concurso se implementó en todos los juegos que se realizaron en el marco del proyecto de investigación con el fin de simplificar la logística y generar unas expectativas comunes en los participantes. La experiencia indica que a los estudiantes los motiva competir por los puntos, aunque en muchos casos el esquema de incentivos terminaba siendo parte del debate final, pues la competencia ayudaba a explicar algunos comportamientos egoístas. Actualmente, cuando el protocolo se usa como estrategia pedagógica en los cursos no se realiza ningún pago monetario, pero es recurrente escuchar que los estudiantes sienten que la competencia es en sí misma un incentivo.

\section{DISCUSIÓN Y RESULTADOS}

Una vez finalizados todos los periodos hay un espacio en el cual se realiza una retroalimentación del juego y se hace una discusión grupal. Este debate busca crear una conexión entre lo práctico y lo teórico, permitiendo la asimilación de los conceptos económicos a partir de 
la experiencia. En este sentido, a continuación, se presenta la forma cómo se diseñó la estrategia pedagógica del debate sobre el mercado de los limones. Es importante aclarar que se siguió en gran medida lo sugerido por Holt y Sherman (1999), y se adaptó y validó en varias pruebas piloto al interior del grupo EMAR. Claramente cada sesión tiene sus particularidades, por lo que se hará énfasis en la forma de moderar el debate, dando un amplio margen para que los estudiantes elaboren sus propias conclusiones.

Para permitir una mayor apropiación de los conceptos, la discusión se organiza a partir de unas preguntas guía. Los participantes comparten su punto de vista para entender los incentivos que los llevaron a tomar ciertas decisiones, teniendo en cuenta que todos los participantes (aleatoriamente) deciden en los dos roles (vendedor y comprador) y pasan por los dos tratamientos. Las preguntas utilizadas para dicha socialización y la discusión se describen a continuación.

Cuando el comprador conoce la calidad del bien, ¿̇cuál crees que es la calidad que más proponen los vendedores? ¿Cuál crees que es el precio promedio que proponen los vendedores? Cuando el comprador no conoce la calidad del bien, ¿̇uál crees que es la calidad que más proponen los vendedores? ¿Cuál crees que es el precio promedio que proponen los vendedores? $\mathrm{El}$ profesor y el personal de apoyo facilitan la discusión, dando la palabra a los participantes y moderando las intervenciones.

Gráfica 1

Promedio de calidades y precios por tratamiento

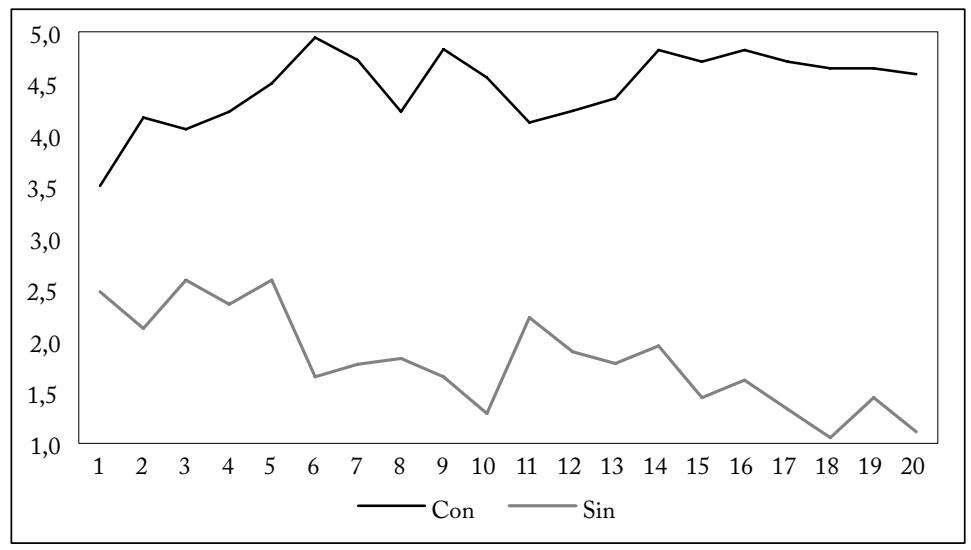

Fuente: elaboración propia.

La discusión comienza típicamente con el resultado más saliente: la dramática caída del precio y la calidad en los periodos sin información 
simétrica. Los estudiantes usualmente son capaces de comprender que cuando los compradores poseen menos información, los vendedores pueden sacar ventaja de la situación y reducir la calidad; entonces los compradores reaccionan protegiéndose a sí mismos. Para el total de la muestra, en el tratamiento con información simétrica la calidad fue de 4,46 y el precio de 7.438,7, en promedio; mientras que sin información simétrica el promedio de la calidad fue de tan solo 1,18 y el precio promedio de 4.734,9. En la gráfica 1 se puede observar que este patrón se mantiene a lo largo de los periodos.

El debate continúa con preguntas que indagan si los compradores o vendedores se benefician de las bajas combinaciones de precio/ calidad, por ejemplo: ${ }_{\zeta}$ Crees que los compradores aceptan menos transacciones cuando no conocen la calidad del bien? Es interesante ver cómo los estudiantes pueden llegar a un consenso sobre los efectos negativos de transar bienes de baja calidad; concluyen que esto perjudica a todos por un menor margen de ganancia y por una mayor tasa de rechazo.

Para esto se debe hacer énfasis en la diferencia entre los valores del comprador y los costos del vendedor. Los estudiantes pueden ver que la calidad 5 es óptima, en el sentido de que crea mejores ganancias para los vendedores y compradores. Como se observa en la gráfica 2 , la aceptación en el tratamiento sin información simétrica cae y se mantiene por debajo en casi todos los periodos (excepto el periodo 2), comparado con el tratamiento donde se puede observar la calidad.

Gráfica 2

Porcentaje de transacciones efectuadas a lo largo de los periodos por tratamiento

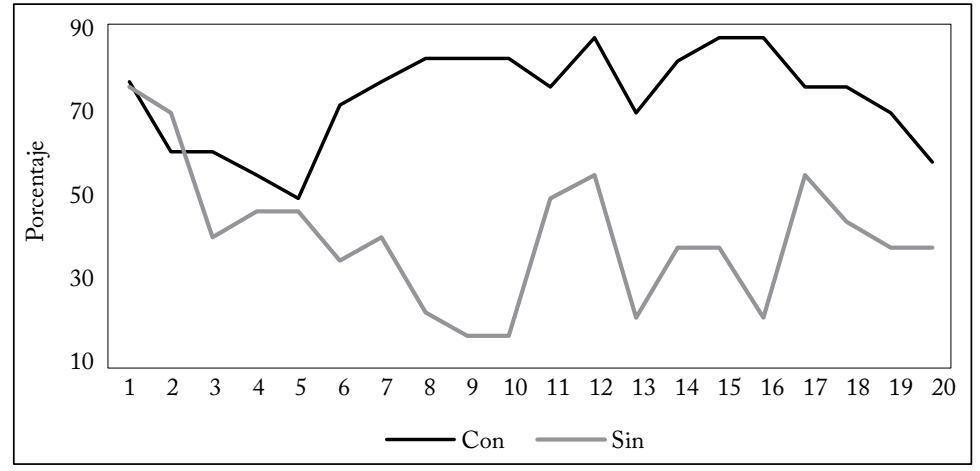

Fuente: elaboración propia.

Por último, se busca que la discusión se enfoque en aquellos factores que puedan remediar las ineficiencias causadas por la asimetría de 
información. Es muy satisfactorio cuando los estudiantes deducen cómo las garantías pueden ayudar a solucionar este problema, protegiendo a los compradores y haciendo su comportamiento defensivo innecesario. En mercados con compras repetidas los vendedores pueden establecer y mantener reputaciones de buena calidad que ellos proveen, y un resultado eficiente puede evolucionar incluso cuando los compradores no pueden observar la calidad antes de la compra.

Estos resultados descriptivos se pueden validar por medio de un análisis de regresión para determinar la significancia estadística. En la cuadro 3 se observa la decisión del vendedor en la variable precio, y la del comprador en la variable aceptación ${ }^{6}$. Lo más interesante es que el precio y la aceptación son significativamente superiores cuando los participantes están en el tratamiento con información sobre la calidad. En efecto, la variable Con calidad tiene un coeficiente positivo y significativo en todas las especificaciones.

Cuadro 3

Análisis de regresión

\begin{tabular}{lcccc}
\hline Variable & \multicolumn{2}{c}{ Precio } & \multicolumn{2}{c}{ Aceptación } \\
\hline Periodo & $(1)$ & $(2)$ & $(3)$ & $(4)$ \\
Reinicio & $-60,089$ & $-70,771$ & $-0,067^{* * *}$ & $-0,070^{* *}$ \\
Con calidad & $-31,199$ & 30,456 & $0,869^{* * *}$ & $0,912^{* * *}$ \\
Mujer & $2.719,737^{* * * *}$ & $2.731,742^{* * *}$ & $1.493^{* * * *}$ & $1.497^{* * *}$ \\
Edad & & $-276,582$ & $-0,102$ \\
Semestre & $51,629^{* * *}$ & & 0,009 \\
Economía & $-81,668$ & & 0,024 \\
Tiempo vendedor & & $464,212^{* *}$ & & $0,469^{* * *}$ \\
Constante & & $-9,626$ & $-0,651$ \\
\hline Estimación de panel con efectos aleatorios. Clúster de sesión. Significancia: ${ }^{*} \mathrm{p}<0,1 ;{ }^{* *} \mathrm{p}<0,05 ;$ \\
* $^{* * *} \mathrm{p}<0,01$ a $^{\mathrm{a}}$ Se refiere al tiempo que tomó el vendedor para tomar la decisión.
\end{tabular}

En términos de la investigación sobre innovación pedagógica, en la fase final del juego se aplicó una encuesta de percepción que permite evidenciar la satisfacción de los estudiantes por participar en el juego. En este sentido, se pidió que respondieran unas preguntas de forma completamente anónima, donde debían, para cada una de las siguientes afirmaciones, marcar el valor que describe de forma más apropiada su situación. Las afirmaciones con su respectiva respuesta promedio (donde $100 \%$ es totalmente de acuerdo) son: 1. En general considero que el juego ha sido muy interesante (91\%); 2. Me gustaria asistir a otros juegos (90\%); 3. Considero que este juego ha sido más entretenido que una clase normal (92\%); 4. Le recomendaría a otra persona que asistiera a estos juegos (94\%).

${ }^{6}$ Las columnas (1) y (2) presentan los resultados de un análisis de datos de panel y las columnas (3) y (4) incluyen las estimaciones de un modelo logit en panel. 
Aunque se ha tenido una buena respuesta por parte de los estudiantes, en este juego y en general en los otros que ha realizado el grupo EMAR, estas preguntas de percepción no están exentas de sesgos, por lo que simplemente muestran que se están consiguiendo resultados positivos en cuanto a la aceptación de esta nueva metodología. En este sentido, el reto para futuras investigaciones es cómo evaluar adecuadamente el aprendizaje que se alcanza con esta técnica. Es importante dejar abierto este punto porque de ninguna manera se quiere afirmar que los juegos buscan reemplazar las clases magistrales y los talleres que fundamentan los conceptos teóricos necesarios para la formación de los economistas. Avanzar en esta dirección dará mayor claridad sobre cómo los juegos en el salón de clase pueden complementar los cursos, y sobre cómo pueden convertirse en una herramienta de divulgación para un público más amplio.

\section{CONCLUSIONES}

En este artículo se ha argumentado que los juegos en el salón de clase son una alternativa pedagógica para el proceso de enseñanza-aprendizaje porque permiten acercar las teorías abstractas a las decisiones reales. Las herramientas de la economía experimental adaptadas para este propósito facilitan la comprensión de los conceptos a través de la experiencia en ambientes digitales controlados. Dado que los estudiantes toman decisiones e interactúan con sus compañeros, desarrollan competencias de forma colaborativa, asumiendo una posición activa y crítica. Adicionalmente, la posibilidad de que estudiantes de semestres superiores se vinculen al grupo de investigación en la modalidad de pasantía permite una apropiación más profunda de los conceptos y las metodologías.

Con respecto a los resultados observados en el juego del mercado de los limones, intuitivamente se esperaría que la balanza se incline a favor del agente con mayor información; en este caso, hacia el vendedor, quien tiene mayor poder de negociación. Pero la interacción muestra una negociación mucho más compleja y enriquecedora en términos pedagógicos. Cuando todos los participantes conocen la calidad de los bienes, el mercado funciona eficientemente y los productos de mejor calidad son los que tienden a ser más transados. Por el contrario, en un entorno de información asimétrica el mercado colapsa, dejando de asignar los recursos eficientemente. Por desconfianza de los compradores, los bienes de menor calidad terminan sacando del mercado a los de mejor calidad. Por lo tanto, este artículo presenta el proceso de validación del protocolo en el laboratorio de economía experimental, 
que puede ser utilizado con fines pedagógicos en la explicación de los efectos de las asimetrías de información en los mercados.

De esta manera, la diferencia que se debe resaltar es que los experimentos tienen como objetivo reunir datos de investigación para contrastar empíricamente una proposición teórica, mientras los juegos en el salón de clase pretenden agregar valor pedagógico en la enseñanza de diferentes disciplinas, como la economía, la ciencia política o la administración de empresas. Actualmente existen diversos centros de investigación con laboratorios de economía experimental, y en Colombia ya existe una comunidad de investigadores formados en esta área que pueden diseñar, adaptar y validar nuevos protocolos experimentales con esta finalidad.

La ventaja del enfoque aquí propuesto es que permite consolidar un equipo de trabajo, es decir, con la realización de una serie de juegos es posible acumular experiencia a manera de semillero. En la medida que se consolida la comunidad de aprendizaje, conformada por estudiantes y profesores, esta estrategia permite la formación para la investigación. Por consiguiente, el reto es diseñar más juegos o adaptar los que se encuentran disponibles en la literatura con el fin de mejorar los contenidos curriculares y las metodologías de enseñanza. Una de las ventajas de contar con un laboratorio es que este facilita la logística. Sin embargo, un laboratorio no es sólo una infraestructura física, sino también un espacio de formación de investigadores, que a través de estos proyectos pueden aumentar la calidad de la educación y modernizar la labor docente.

\section{REFERENCIAS BIBLIOGRÁFICAS}

1. Akerlof, G. "The market for 'lemons': Quality uncertainty and the market mechanism", The Quarterly Journal of Economics 84, 3, 1970, pp. 488-500.

2. Becker, W. y M. Watts. "Teaching methods in U. S. undergraduate economics courses", Journal of Economic Education 32, 3, 2001, pp. 269-279.

3. Brañas-G., P, ed. Economía experimental y del comportamiento, Madrid, Antoni Bosh, 2011.

4. Brandts, J. "La economía experimental y la economía del comportamiento", Filosofía y economía: una mirada metodológica, Madrid, Trotta, 2007.

5. Capra, M.; J. Goeree et al. "Predation, asymmetric information and strategic behavior in the classroom: An experimental approach to the teaching of industrial organization", International Journal of Industrial Organization 18, 1, 2000, pp. 205-225. 
6. Emerson, T. "Anyone? Anyone? A guide to submissions on classroom experiments", The Journal of Economic Education 45, 2, 2014, pp. 174-179.

7. Fischbacher, U. "z-Tree: Zurich toolbox for ready-made economic experiments", Experimental Economics 10, 2, 2007, pp. 171-178.

8. Friedman, D. y S. Sunder. Experimental methods: A primer for economists, Nueva York, Cambridge University Press, 1994.

9. Friedman, D. y A. Cassar, Economic lab. An intensive course in experimental economics, Londres, Routledge, 2005.

10. García, M. "Lo que aprendí en Fundamentos de Economía”, Cuadernos de Economía 23, 40, 2004, pp. 231-243.

11. Holt, C. "Classroom games: Trading in a pit market", Journal of Economic Perspectives 10, 1, 1996, pp.193-203.

12. Holt, C. Markets, games, E strategic behavior, Nueva York, Pearson, 2007.

13. Holt, C. y R. Sherman. "Classroom games: A market for lemons", Journal of Economic Perspectives 13, 1, 1999, pp. 205-214.

14. Lora, E. y H. Nopo. "La formación de los economistas en América Latina”, Revista de Análisis Económico 24, 2, 2009, pp. 65-93.

15. Montenegro, Á. Introducción a la economía experimental, Bogotá, Uniandes, 1995.

16. Muñoz, J.; S. Aparicio y M. Mesa. “¿Qué la economía no es un juego? Evidencia del uso de economía experimental en cursos de introducción a la Economía”, Ecos de Economía 15, 33, 2011, pp. 185-207.

17. Palacio, L. y D. Parra. "Economía experimental: un panorama general”, Revista Lebret 4, 2012, pp. 277-294.

18. Salazar, A. "La economía experimental como aprendizaje significativo en el aula”, Revista Páginas 88, 2010, pp. 41-47.

19. Saravia, I. y M. Vesga. "Juegos en el salón de clase: el mercado de los limones”, Bucaramanga, Universidad Industrial de Santander, 2015.

20. Sarnikar, S. What can behavioral economic teach us about teaching economics?, Nueva York, Palgrave, 2015.

21. Smith, V. “¿Qué es la economía experimental?”, Apuntes del cenes 25, 39, 2005, pp. 7-16.

22. Stiglitz, J. "La información y el cambio en el paradigma de la ciencia económica", Revista Asturiana de Economía 25, 2002, pp. 95-164.

23. Wolf, J y M. Myerscough. "Reputations in markets with asymmetric information: A classroom game", The Journal of Economic Education 38, 4, 2007, pp. 393-405. 